\title{
Hyperbolic theories of dissipation: Why and when do we need them?
}

\author{
Luis Herrera* \\ Escuela de Física, Facultad de Ciencias, \\ Universidad Central de Venezuela, Caracas, Venezuela. \\ and \\ Diego Pavón ${ }^{\dagger}$ \\ Departamento de Física, Facultad de Ciencias, \\ Edificio Cc, Universidad Autónoma de Barcelona, \\ 08193 Bellaterra, Spain.
}

\begin{abstract}
We illustrate and emphasize the relevance of hyperbolic theories of dissipation in different physical scenarios. Particular attention is paid to self-gravitating systems where the relaxation time may become large enough as to require a description of the transient regime. It is argued that even outside that regime, hyperbolic theories may be needed to provide an accurate description of dissipative processes.
\end{abstract}

\footnotetext{
*Postal address: Apartado 80793, Caracas 1080A, Venezuela; E-mail address: laherrera@telcel.net.ve

${ }^{\dagger}$ E-mail address: diego@ulises.uab.es
} 


\section{Introduction}

In the description of the evolution of any physical system, it is mandatory to evaluate, as accurateely as possible, the order of magnitude of different characteristic time scales, since their relationship with the time scale of observation (the time during which we assume our description of the system to be valid) will determine, along with the relevant equations, the evolution pattern. Take a forced damped harmonic oscillator and consider its motion on a time scale much larger than both the damping time and the period of the forced oscillation. Then, what one observes is just a harmonic motion. Had we observed the system on a time scale of the order of (or smaller) than the damping time, the transient regime would have become apparent. This is rather general and of a very relevant interest when dealing with dissipative systems. It is our purpose here, by means of examples and arguments related to a wide class of phenomena, to emphasize the convenience of resorting to hyperbolic theories when dissipative processes, either outside the steady-state regime or when the observation time is of the order or shorther than some characteristic time of the system, are under consideration. Furthermore, as it will be mentioned below, transient phenomena may affect the way in which the system leaves the equilibrium, thereby affecting the future of the system even for time scales much larger than the relaxation time.

\section{The rationale behind hyperbolic theories}

Parabolic theories of dissipative phenomena have long and a venerable history and proved very useful especially in the steady-state regime [1]. They exhibit however some undesirable features, such as acausality (see e.g., [2], [3]), that prompted the formulation of hyperbolic theories of dissipation to get rid of them [4], [3]. This was achieved at the price of extending the set of field variables by including the dissipative fluxes (heat current, non-equilibrium stresses and so on) at the same footing as the classical ones (energy densities, equilibrium pressures, etc), thereby giving rise to a set of more physically satisfactory (as they much better conform with experiments) but involved theories from the mathematical point of view. These theories have the additional advantage of being backed by statistical fluctuation theory, kinetic theory of gases (Grad's 13-moment approximation), information theory and correlated random walks (at least in the version of Jou et al.) [3].

A key quantity in these theories is the relaxation time $\tau$ of the corresponding dissipative process. This positive-definite quantity has a distinct physical meaning, namely the time taken by the system to return spontaneously to the steady state (whether of thermodynamic equilibrium or not) after it has been suddenly removed from it. It is, however, somehow connected to the mean collision time $t_{c}$ of the particles responsible for the dissipative process, ofentimes erroneously identified with it. In principle they are different since $\tau$ is (conceptually and many times in practice) a macroscopic time, although in some instances it may correspond just to a few $t_{c}$. No general formula linking $\tau$ and 
$t_{c}$ exists, their relationship depends in each case on the system under consideration. As mentioned above, it is therefore appropriate to interpret $\tau$ as the time taken by the corresponding dissipative flow to relax to its steady value.

Thus, it is well known that the classical Fourier law for heat current,

$$
\vec{q}=-\kappa \vec{\nabla} T
$$

with $\kappa$ the heat conductivity of the fluid, leads to a parabolic equation for temperature (diffusion equation),

$$
\frac{\partial T}{\partial t}=\chi \nabla^{2} T \quad\left(\chi \equiv \frac{\kappa}{\rho c_{p}}\right)
$$

(where $\chi, \rho$ and $c_{p}$ are the diffusivity, density and specific heat at constant pressure, respectively), which does not forecast propagation of perturbations along characteristic causal light-cones (see [3], [5], [6], [7] and references therein). That is to say, perturbations propagate with infinite speed. This non-causal behavior is easily visualized by taking a look at the thermal conduction in an infinite one dimensional medium (see e.g. [1], [8]). Assuming that the temperature of the line is zero for $t<0$, and putting a heat source at $x=x_{0}$ when $t=0$, the temperature profile for $t>0$ is given by

$$
T \propto \frac{1}{\sqrt{t}} \exp \left[-\frac{\left(x-x_{0}\right)^{2}}{t}\right],
$$

implying that for $t=0 \Longrightarrow T=\delta\left(x-x_{0}\right)$, and for $t=\tilde{t}>0 \Longrightarrow T \neq 0 \forall x$.

In other words, the presence of a heat source at $x_{0}$ is instantaneously felt by all observers on the line, no matter how far away from $x_{0}$ they happen to be. The origin of this behavior can be traced to the parabolic character of Fourier's law, which implies that the heat flow starts (vanishes) simultaneously with the appearance (disappearance) of a temperature gradient. Although $\tau$ is very small for phonon-electron, and phonon-phonon interaction at room temperature $\left(\mathcal{O}\left(10^{-11}\right)\right.$ and $\mathcal{O}\left(10^{-13}\right)$ seconds, respectively [9]), neglecting it is the source of difficulties, and in some cases a bad approximation as for example in superfluid Helium [10], and degenerate stars where thermal conduction is dominated by electrons -see [3], [5], [11], for further examples.

In order to overcome this problem Cattaneo and (independently) Vernotte by using the relaxation time approximation to Boltzmann equation for a simple gas derived a generalization of Fourier's law, namely [12]

$$
\tau \frac{\partial \vec{q}}{\partial t}+\vec{q}=-\kappa \vec{\nabla} T .
$$

This expression (known as Cattaneo-Vernotte's equation) leads to a hyperbolic equation for the temperature (telegraph equation)

$$
\tau \frac{\partial^{2} T}{\partial t^{2}}+\frac{\partial T}{\partial t}=\chi \nabla^{2} T
$$


which describes the propagation of thermal signals with a finite speed

$$
v=\sqrt{\chi / \tau}
$$

This diverges only if the unphysical asumption of setting $\tau$ to zero is made.

It is worth mentioning that a simple random walk analysis of transport processes naturally leads to telegraph equation, not to the diffusion equation -see e.g. [13]. Again, the latter is obtained only if one neglects the second derivative term.

It is instructive to write (雨) in the equivalent integral form

$$
\vec{q}=-\frac{\kappa}{\tau} \int_{-\infty}^{t} \exp \left[-\frac{\left(t-t^{\prime}\right)}{\tau}\right] \cdot \vec{\nabla} T\left(\vec{x}, t^{\prime}\right) d t^{\prime}
$$

which in turn is a particular case of the more general expression

$$
\vec{q}=-\int_{-\infty}^{t} Q\left(t-t^{\prime}\right) \vec{\nabla} T\left(\vec{x}, t^{\prime}\right) d t^{\prime}
$$

The physical meaning of the kernel $Q\left(t-t^{\prime}\right)$ becomes obvious by observing that

$$
\begin{array}{cccc}
\text { for } \quad Q=\kappa \delta\left(t-t^{\prime}\right) & \Longrightarrow & \vec{q}=-\kappa \vec{\nabla} T & \text { (Fourier) } \\
\text { for } \quad Q=\text { constant } & \Longrightarrow & \frac{\partial^{2} T}{\partial t^{2}}=\chi \nabla^{2} T,
\end{array}
$$

i.e., $Q$ describes the thermal memory of the material by assigning different weights to temperature gradients at different moments in the past. The Fourier law corresponds to a zero-memory material (the only relevant temperature gradient is the "last" one, i.e., the one simultaneous with the appearance of $\vec{q})$. By contrast the infinite memory case (with $Q=$ constant) leads to an undamped wave. Somewhere in the middle is the Cattaneo-Vernotte equation, for which all temperature gradients contribute to $\vec{q}$, but their relevance goes down as we move to the past.

From these comments it should be clear that different classes of dissipative systems may be described by different kernels. The one corresponding to (4) being suitable for the description of a restricted subclass of phenomena.

Obviously, when studying transient regimes, i.e., the evolution from a steadystate situation to a new one, $\tau$ cannot be neglected. In fact, leaving aside that parabolic theories are necessarily non-causal, it is obvious that whenever the time scale of the problem under consideration becomes of the order of (or smaller) than the relaxation time, the latter cannot be ignored. It is common sense what is at stake here: neglecting the relaxation time ammounts -in this situation- to disregarding the whole problem under consideration. 


\section{Hyperbolic versus parabolic}

According to a basic assumption underlying the disposal of hyperbolic dissipative theories, dissipative processes with relaxation times comparable to the characteristic time of the system are out of the hydrodynamic regime. However, the concept of hydrodynamic regime involves the ratio between the mean free path of fluid particles and the characteristic length of the system. When this ratio is lower that unity, the fluid is within the hydrodynamic regime. When it is larger than unity, the regime becomes Knudsen's. In the latter case the fluid is no longer a continuum and even hyperbolic theories cease to be realiable.

Therefore that assumption can be valid only if the particles making up the fluid are the same ones that transport the heat. However, this is (almost?) never the case. Specifically, for a neutron star, $\tau$ is of the order of the scattering time between electrons (which carry the heat) but this fact is not an obstacle (no matter how large the mean free path of these electrons may be) to consider the neutron star as formed by a Fermi fluid of degenerate neutrons. The same is true for the second sound in superfluid Helium and solids, and for almost any ordinary fluid. In brief, the hydrodynamic regime refers to fluid particles that not necessarily (and as a matter of fact, almost never) transport the heat. Therefore large relaxation times (large mean free paths of particles involved in heat transport) does not imply a departure from the hydrodynamic regime (this fact has been streseed before [14], but it is usually overlooked).

However, even in the case that the particles making up the fluid are responsible of the dissipative process, it is not "always" valid to take for granted that $\tau$ and $t_{c}$ are of the same order (se e.g. [15], [16]), or what comes to the same that the dimensionless quantity $\Gamma \equiv\left(\tau c_{s} / L\right)^{2}$ is negligible in all instances -here $c_{s}$ stands for the adiabatic speed of sound in the fluid under consideration and $L$ the characteristic length of the system. That assumption would be right if $\tau$ were always comparable to $t_{c}$ and $L$ always "large", but there are, however, important situations in which $\tau \gg t_{c}$, and $L$ "small" although still large enough to justify a macroscopic description. For tiny semiconductor pieces of about $10^{-4}$ $\mathrm{cm}$ in size, used in common electronic devices submitted to high electric fields, the above dimensionless combination (with $\tau \sim 10^{-10} \mathrm{sec}, c_{s} \sim 10^{7} \mathrm{~cm} / \mathrm{sec}$ [17]) can easily be of the order of unity. In ultrasound propagation as well as light-scattering experiments in gases and neutron-scattering in liquids the relevant length is no longer the system size, but the wavelenght $\lambda$ which is usually much smaller than $L$ [18], 19]. Because of this, hyperbolic theories may bear some importance in the study of nanoparticles and quantum dots. Likewise in polymeric fluids relaxation times are related to the internal configurational degres of freedom and so much longer than $t_{c}$ (in fact they are in the range of the minutes), and $c_{s} \sim 10^{5} \mathrm{~cm} / \mathrm{sec}$, thereby $\Gamma \sim \mathcal{O}(1)$. In the degenerate core of aged stars the thermal relaxation time can be as high as 1 second [20]. Assuming the radius of the core of about $10^{-2}$ times the solar radius, one has $\Gamma \sim \mathcal{O}(1)$ again. Fully ionized plasmas exhibit a collisionless regime (Vlasov regime) for which the parabolic hydrodynamics predicts a plasmon dispersion relation at variance with the microscopic results; the latter agree, however, with the hyperbolic hydrodynamic approach [21]. Think for instance of some syrup 
fluid flowing under a imposed shear stress, and imagine that the shear is suddenly switched off. This liquid will come to a halt only after a much longer time $(\tau)$ than the collision time between its constituent particles has elapsed.

The fact that $\tau$ can quantitatively greatly differ from $t_{c}$ is most dramatically suggested by the matter-radiation decoupling in the early universe. In a recent paper Pavón and Sussman [22] by using the Lemaitre-Tolmann-Bondi metric [23] along with the hyperbolic transport equation for the shear-stress

$$
\tau \dot{\Pi}_{c d} h_{a}^{c} h_{b}^{d}+\Pi_{a b}\left[1+\frac{1}{2} T \eta\left(\frac{\tau}{T \eta} u^{c}\right)_{; c}\right]+2 \eta \sigma_{a b}=0, \quad \text { with } \quad \eta=\frac{4}{5} p \tau,
$$

showed that the relaxation time of shear viscosity results several ordersof magnitude larger than the Thomson collision time between photons and electrons for most of the radiative era, i.e., in the temperature range $10^{3}<T<10^{6}$ Kelvin. (In above expression $\Pi_{a b}$ denotes the shear-stress tensor arising from the matter-radiation interaction, $\eta$ is the transport coefficient, and $\sigma_{a b}$ the symmetric trace-free part of the gradient of the four-velocity, and $p$ the radiation pressure. In this scenario $t_{c}$ is obtained by introducing the Thomson cross-section in Saha's formula.

Many other examples could be added but we do not mean to be exhaustive.

Even in the steady regime the descriptions offered by parabolic and hyperbolic theories do not necessarily coincide. The differences between them in such a situation arise from (i) the presence of $\tau$ in terms that couple the vorticity to the heat flux and shear stresses. These may be large even in steady states (e.g. rotating stars). There are also other acceleration coupling terms to bulk and shear stresses and heat flux. The coefficientsfor these vanish in parabolic theories, and they could be large even in the steady state. (ii) From the convective part of the time derivative (which are not negligible in the presence of large spatial gradients). (iii) From modifications in the equations of state due to the presence of dissipative fluxes [3].

However, it is precisely before the establishment of the steady regime that both types of theories differ more importantly. It is well-known (see [3], [5], [11], [24]) that a variety of physical processes take place on time scales of the order of (or even smaller) than the corresponding relaxation time, which as was stressed above does not imply that the system is out of hydrodynamic regime. Therefore if one wishes to study a dissipative process for times shorter than $\tau$, it is mandatory to resort to a hyperbolic theory which is a more accurate macroscopic approximation to the underlying kinetic description.

In the particular case of neutron star matter, it appears that the relaxation time may indeed be of the order of magnitude of the characteristic time of some physical relevant processes. Thus, from (6) one has

$$
\tau=\frac{\kappa}{v^{2} \rho c_{p}}
$$

If the heat conductivity is dominated by electrons (as is the case of a neutron star), then we can adopt the expression 25] 


$$
\kappa \approx 10^{23}\left[\rho / 10^{14} \mathrm{~g} \quad \mathrm{~cm}^{-3}\right]\left[10^{8} \mathrm{~K} / T\right] \mathrm{erg} \quad \mathrm{s}^{-1} \mathrm{~cm}^{-1} \mathrm{~K}^{-1}
$$

and for the specific heat $c_{p}=\beta T / M$, where $\beta$ (which is model dependent) is given by [26] $\beta \approx 10^{29} \mathrm{erg} / \mathrm{K}^{2}$ and $M$ denotes the total mass. Feeding back these expressions in (11) we get

$$
\tau \approx \frac{10^{20}}{\left[T^{2}\right]\left[v^{2}\right]} \mathrm{s},
$$

where $[T]$ and $[v]$ denote the numerical values of the temperature and the velocity of the thermal wave in Kelvin and $\mathrm{cm} / \mathrm{s}$, respectively. We have further assumed $\rho \approx 10^{14} \mathrm{~g} \mathrm{~cm}^{-3}$ and approximately $10 \mathrm{Km}$ for the radius of the degenerate core. In this case, assuming for $v$ the speed of light in vacuum, for temperatures of the order of $[T] \approx 10^{2}$ Kelvin -a certainly low value that corresponds to the latest phases in the evolution of a neutron star (see [26])- we get $\tau \approx 10^{-5}$ seconds.

However, for more reasonable values of $v$, such as $10^{3} \mathrm{~cm} / \mathrm{s}$, corresponding to the speed of second sound in superfluid helium, and for temperatures of the order of $10^{9}$ Kelvin, one obtains $\tau \approx 10^{-4}$ seconds. Whereas for $T \approx 10^{6}$ Kelvin, the relaxation time may be found as large as $\tau \approx 10^{2}$ seconds. Therefore physical process on a time scale of the order (or smaller) than above belong to the transient regime and require a hyperbolic theory.

Another astrophysical phenomenom where the effects of the transient regime can be felt is the double peaked temporal luminosity profile observed from $\mathrm{x}^{-}$ ray bursters in star explosions, such as the one reported by Hoffman et al. [27]. These authors observed a $\mathrm{x}$-ray burster showing a precursor peak, that lasted about 4 seconds, neatly separated from the main event -which lasted above 1000 seconds. While the analysis of elastic photon diffusion through a cloud of ionized plasma of modest optical depth $\tau_{*}$ via parabolic theories just predicts the convential diffusion structure, the hyperbolic theory predicts a well defined peak for times shorter than $t_{D} \sim \tau_{*}^{2} t_{c} / 2$ and a diffusive behavior for $t>t_{D}$ [28]. This kind of event is reminiscent of laser induced heat pulses in solids where the double structure is evident [29]. Again hyperbolic theories successfully deal with such situations while parabolic do not [3].

Finally, it is worth mentioning that relaxation time appears to be quite relevant in the outcome of gravitational collapse as many numerical and analytical calculations indicate (see [24], [30] and references therein). In particular it has been shown that, for otherwise identical boundary and initial conditions, larger values of $\tau$ imply more flattened and long lasting pulses of emission, thereby affecting the evolution of the object.

\section{Beyond the transient regime}

So far we have seen that only for times larger than $\tau$ it is sensible to resort to a parabolic theory (modulo that the spatial gradients are not so large that 
the convective part of the time derivative becomes important, and that the fluxes and coupling terms remain safely small). However, even in these cases, it should be kept in mind that the way a system leaves the equilibrium may be very sensitive to the relaxation time.

Indeed, it has been shown [31] that after the fluid leaves the equilibrium, on a time scale of the order of relaxation time, the effective inertial mass density of a dissipative fluid reduces by a factor that depends on the dissipative variables. By "effective inertial mass density" (EIMD) we mean the factor of proportionality between the applied three-force density and the corresponding proper acceleration (i.e., the three-acceleration measured in the instantaneous rest frame). The expression for the EIMD contains a contribution from dissipative variables which reduces its value with respect to the equilibrium situation.

Specifically, it has been shown that just after leaving the equilibrium on a time scale of the order of $\tau$ the EIMD becomes (in relativistic units)

$$
\Lambda=(\rho+p)(1-\alpha),
$$

with

$$
\alpha=\frac{\kappa T}{\tau(\rho+p)}
$$

giving rise, in principle, to the possibility of a vanishing $\Lambda$ (i.e., $\alpha=1$ ) or even negative $(\alpha>1)$. Such effect may be present in any dissipative fluid (either self-gravitating or not) 32.

In order to evaluate $\alpha$, let us turn back to coventional units. Assuming for simplicity $\rho+p \approx 2 \rho$, we obtain

$$
\alpha=\frac{\kappa T}{\tau(\rho+p)} \approx \frac{[\kappa][T]}{[\tau][\rho]} \times 10^{-42}
$$

where $[\kappa],[T],[\tau],[\rho]$ denote the numerical values of these quantities in c.g.s units.

Obviously, except for extremely high values of $\kappa$ and $T, \alpha$ will be a much less than unity. Observe, in this connection, that although the smaller $\tau$, the larger $\alpha$, this is of little consequence since (14) is valid only on a time scale of the order of $\tau$. Therefore, a decreasing of $\Lambda$ with physically relevant impact requires values of (16) close to unity, due to large values of $\kappa$ and $T$ but non-negligible values of $\tau$.

A possible scenario where $\alpha$ may decrease substantially (for non-negligible values of $\tau$ ) might be provided by a pre-supernova event. At the last stages of massive star evolution, the decreasing of the opacity of the fluid, from very high values preventing the diffusion of photons and neutrinos (trapping [33]), to smaller values, gives rise to a radiative heat conduction. Upon these conditions both $\kappa$ and $T$ could become sufficiently large as to imply a substantial increase of $\alpha$. Indeed, the values suggested in [34] $\left([\kappa] \approx 10^{37} ;[T] \approx 10^{13} ;[\tau] \approx 10^{-4}\right.$; $[\rho] \approx 10^{12}$, in c.g.s. units) lead to $\alpha \sim \mathcal{O}(1)$. The obvious consequence would be to enhance the efficiency of whatever expansion mechanism, of the central 
core, because of the decreasing of $\Lambda$. Thus, it becomes clear that the value of $\alpha$ in the transient regime may critically affect the subsequent evolution of the system. Therefore it may be said that the future of the system at time scales much longer than the relaxation time (once the steady state is reached), may also critically depend on $\tau$.

\section{Concluding remarks}

It has been argued that the infinite speeds of propagation predicted by parabolic theories is just a consequence of using them outside their range of applicability which is limited by the discrete nature of matter [13]. In this connection it should be noted that hyperbolic theories also share this constraint but they naturally predict speeds in good agreement with experiments. Therefore one is led to conclude that hyperbolic theories have a range of aplicability much wider than parabolic theories.

Aside from $\tau$ hyperbolic theories introduce a certain number of new parameters that couple the different dissipative fluxes. In spite of this being rather natural (as these theories are designed to explain more complex phenomena than parabolic theories do), this feature has been under unduly criticism as though these quantities were "free parameters" that one could choose at will [15]. This is not the case. On the one hand, their number gets severely reduced when one realizes that they are interrelated. For instance, for an ideal gas under heat flux and bulk and shear stresses the parameters entering the transport equations of second order hyperbolic theory (equations (2.31)-(2.33) in [3.a]) are linked by the six equations (3.44)-(3.45) in [3.a]. On the other hand, these parameters are restricted by the convexity of the entropy function, and can be calculated explicitly with the help of kinetic theory, or fluctuation theory, such as in the case of a radiative fluid [35].

In summary, although parabolic theories have proved very useful for many practical purposes, they appear to fail hopelessly in a number of well-known instances (such as transient regimes). By contrast, hyperbolic theories successfully predict the experimental results, and so, in these regimes hyperbolic theories happen to be more reliable. In the steady-state (under the conditions mentioned above) and for times exceeding $\tau$ both theories converge. Before closing we would like to emphasize we are not advocating to stop using parabolic transport equations. Our aim is simply to stress the convenience of using hyperbolic transport equations there and when parabolic theories either fail or the problem under consideration happens to lie outside their range of applicability.

We hope this paper will help to convince the reader that hyperbolic theories are indeed of not mere academic interest and it would not be wise to dispense of them.

\section{Acknowledgements}

The authors are indebted to David Jou for reading the manuscript and helpful remarks. This work has been partially supported by the Spanish Ministry of 
Science and Technology under grants BFM 2000-C-03-01 and 2000-1322.

\section{References}

[1] A.L. Fetter and J.D. Walecka, Theoretical Mechanics of Particles and Continua (MacGraw Hill, N.Y., 1980).

[2] W.A. Hiscock and L. Lindblom, Ann. Phys. (N.Y.) 151, 466 (1983); ibid Contemporary Mathematics 71, 181 (1988).

[3] D. Jou, J. Casas-Vázquez and G. Lebon, Extended Irreversible Thermodynamics, second edition (Springer-Verlag, Berlin, 1996); D. Jou, J. CasasVázquez and G. Lebon, Rep. Prog. Phys. 51, 1104 (1988); ibid 62, 1035 (1999).

[4] I. Müller, Arch. Rat. Mech 34, 259 (1969); W. Israel, Ann. Phys. (N.Y.) 100, 310 (1976); W.G. Dixon, Special Relativity: The Foundations of Macroscopic Physics (Cambridge University Press, Cambridge 1978); W. Israel and J. Stewart, Ann. Phys. (N.Y.) 118, 341 (1979); D. Pavón, D. Jou and J. Casas-Vázquez, J. Phys. A. 13, L77 (1980); D. Pavón, D. Jou and J. Casas-Vázquez, Ann. Inst. H. Poincaré A 36, 79 (1982), I. S. Liu, I. Müller and T. Ruggeri, Ann. Phys. (N.Y.) 169, 191 (1986); I. Müller and T. Ruggeri, Rational Extended Thermodynamics (Springer-Verlag, Berlin, 1998).

[5] D. Joseph and L. Preziosi, Rev. Mod. Phys. 61, 41 (1989); ibid 62, 375 (1990).

[6] R. Maartens, in Proceedings of the Hanno Rund Conference on Relativity and Thermodynamics, ed. S.D. Maharaj (1996), [astro-ph/9609119].

[7] D. Y. Tzou, Macro-to Microscale Heat Transfer: The Lagging Behavior (Taylor \& Francis, Washington, DC, 1996).

[8] L. Landau and E. Lifshitz, Fluid Mechanics (Addison-Wesley, Reading, 1959).

[9] R. Peierls, Quantum Theory of Solids (Oxford University Press, London, 1955).

[10] V. Peshkov, J. Phys. 8, 381, (1944); W. Band and L. Meyer, Phys. Rev. 73, 226 (1948).

[11] A. Anile, D. Pavón and V. Romano, "The case for hyperbolic theories of dissipation in relativistic fluids" (1998) [gr-qc/9810014; D. Pavón, in Relativity and Gravitation in General, ed. J. Martín et al. (World Scientific, Singapore, 1999).

[12] C. Cattaneo, Atti. Semin. Mat. Fis. Univ. Modena 3, 3 (1948); P. Vernotte, Compt. R. Acad. Sci. Paris 246, 3154 (1958). 
[13] H.D. Weymann, Am. J. Phys. 35, 488 (1967).

[14] L.Herrera and N.O. Santos, Monthly Not. R. Astr. Soc. 287, 161 (1997).

[15] R. Geroch, J. Math. Phys. 36, 4226 (1995).

[16] L. Lindblom, Ann. Phys. (N.Y.) 247, 1 (1996).

[17] A.M. Anile and O. Muscato, Phys. Rev. B 51, 16728 (1995).

[18] W. Weiss and I. Müller, Continuum Mech. Thermodyn. 7, 123 (1995).

[19] J.R.D. Copley and S.W. Lovesey, Rep. Progr. Phys. 38, 461 (1975).

[20] M. Harwit, Astrophysical Concepts (Springer-Verlag, Berlin, 1998).

[21] I.V. Tokatly and D. Pankratov, Phys. Rev. B 62, 2759 (2000).

[22] D. Pavón and R.A. Sussman, Class. Quantum Grav. 18, 1625 (2001).

[23] A. Krasiński, Inhomogeneous Cosmological Models (CUP, Cambridge, 1997).

[24] A. Di Prisco, L. Herrera and M. Esculpi, Class. Quantum Grav. 13, 1053 (1996).

[25] N. Flowers and N. Itoh, Astrophys. J., 230847 (1979); Astrophys. J., 250, 750 (1981).

[26] N. Shibazaki and F. Lamb, Astrophys. J. 346, 808 (1989).

[27] J. A. Hoffman, W.G.H. Lewis, J. Doty, J.G. Jernigan, M. Haney and J.A. Richardson, Astrophys. J. Lett. 221, L57 (1978).

[28] A.M. Schweizer, Astron. Astrophys. 152, 79 (1985).

[29] W. Dreyer and H. Struchtrup, Continuum Mech. Thermodyn. 5, 3 (1993).

[30] A. Di Prisco et al. Gen. Rel. Grav. 29, 1391 (1997); M. Govender, S. Maharaj and R. Maartens, Class. Quantum Grav. 15, 323 (1998); M. Govender, R. Maartens and S. Maharaj, Phys.Lett.A, 283, 71 (2001); S. Wagh et al., Class. Quantum Grav. 18, 2147 (2001).

[31] L. Herrera, A. Di Prisco, J. Hernández-Pastora, J. Martín and J. Martínez, Class. Quantum Grav. 14, 223 (1997); L. Herrera and J. Martínez, Class. Quantum Grav. 15, 407 (1998).

[32] L. Herrera "On the efffective inertial mass density of a dissipative fluid" (2001) preprint.

[33] W. Arnett, Astrophys. J. 218, 815 (1977).

[34] J. Martínez, Phys. Rev. D 53, 6921 (1996).

[35] D. Jou and D. Pavón, Astrophys. J. 291, 447 (1985); N. Udey and W. Israel, Mon. Not. R. Astr. Soc. 199, 1137 (1982). 